Baumgarten, Peter, und Wolfgang Múckl, Wirtschaftspolitische Zielkonfikte in der Bundesrepublik Deutschland. Eine theoretische und empirische Untersuchung. Mit $9 \mathrm{Abb}$. u. 12 Tab. (Institut für angewandte Wirtschaftsforschung, Schriftenreihe: Bd. I2.) Tübingen I969. J. C. B. Mohr (Paul Siebeck). IX, 193 S.

Lehmann, Hermann, Grenznutzentheorie. Geschichte und Analyse eines bürgerlichen ökonomischen Lehrsystems. Berlin 1968. Dietz Verlag. $427 \mathrm{~S}$.

Meerhaege, M. A. G. van, Price Theory and Price Policy. London 1969. Longmans, Green \& Co. X, $109 \mathrm{~S}$.

Morishima, Michio, Theory of Economic Growth. London 1969. Oxford University Press. IX, 3 ro S.

Woll, Artur, Allgemeine Volkswirtschaftslehre. (Vahlens Handbucher der Wirtschaftsund Sozialwissenschaften.) Berlin u. Frankfurt a. M. 1969. Franz Vahlen. XVII, $413 \mathrm{~S}$.

\title{
II. Allgemeine und angewandte Statistik
}

Schonfeld, Peter, Methoden der Okonometrie. Bd. I: Lineare Regressionsmodelle. (Vahlens Handbücher der Wirtschafts- und Sozialwissenschaften.) Berlin u. Frankfurt a. M. 1969. Franz Vahlen. XI, 205 S.

\section{Weltwirtschaft und Wirtschaft einzelner Länder und Gebiete}

\section{Weltwirtschaft}

Johnson, Harry G., Economic Policies Towards Less Developed Countries. London 1967. George Allen \& Unwin. XVI, 279 S.

Seit vielen Jahren ist eine rege Diskussion darïber im Gange, auf welche Weise die gegenwärtigen internationalen Wirtschaftsbeziehungen zwischen den Entwicklungsländern und den Industrieländern umgestaltet werden müßten, damit durch sie die Entwicklung in den Entwicklungsländern stärker als bisher gefördert wird. Für diese Auseinandersetzungen kommt es entscheidend auf die Möglichkeit an, die Wirkungen bestimmter Maßnahmen abzuschätzen. Trotz aller Anstrengungen ist unser empirisches Wissen in dieser Hinsicht noch verhältnismäßig gering. Es besteht auch heute noch keine umfassende empirische Grundlage für die Gestaltung der internationalen Wirtschaftsbeziehungen zwischen den Entwicklungsländern und den Industrieländern. Notwendigerweise treten daher Werturteile stärker als für eine sachliche Diskussion zuträglich ist in den Vordergrund.

Im vorliegenden Buch bemüht sich Johnson zwar sehr, seine Ausführungen durch empirische Schätzungen zu untermauern, doch muß sein Vorgehen lückenhaft bleiben; die Schätzungen sollten teilweise wohl mehr als Illustrationen angesehen werden. So liegt das Hauptgewicht des Buches auf theoretischen Deduktionen.

Grundlage für Johnsons Überlegungen zur Frage einer empfehlenswerten Handels- und Entwicklungspolitik der Industrieländer, insbesondere der Vereinigten Staaten, an die sich das Buch vor allem wendet, ist die neo- 